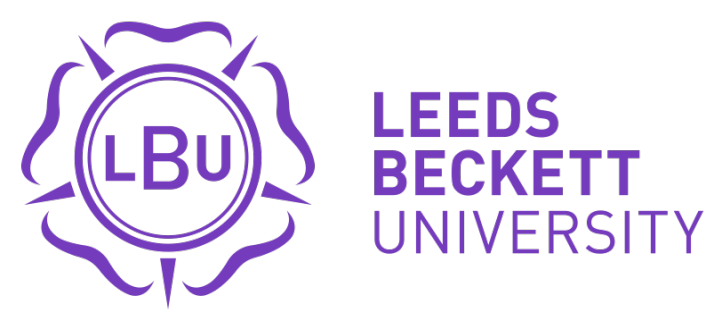

Citation:

Kelley, R and Surr, C and Griffiths, A and Ashley, L and Procter, A (2021) Providing cancer treatment and care to people living with cancer and dementia: challenges and research-based recommendations. Cancer Nursing Practice. ISSN 1475-4266 DOI: https://doi.org/10.7748/cnp.2021.e1754

Link to Leeds Beckett Repository record:

https://eprints.leedsbeckett.ac.uk/id/eprint/7537/

Document Version:

Article (Accepted Version)

This is an Accepted Manuscript of an article published by RCN Publishing in Cancer Nursing Practice on 15th February 2021, available online: http://doi.org/10.7748/cnp.2021.e1754

The aim of the Leeds Beckett Repository is to provide open access to our research, as required by funder policies and permitted by publishers and copyright law.

The Leeds Beckett repository holds a wide range of publications, each of which has been checked for copyright and the relevant embargo period has been applied by the Research Services team.

We operate on a standard take-down policy. If you are the author or publisher of an output and you would like it removed from the repository, please contact us and we will investigate on a case-by-case basis.

Each thesis in the repository has been cleared where necessary by the author for third party copyright. If you would like a thesis to be removed from the repository or believe there is an issue with copyright, please contact us on openaccess@leedsbeckett.ac.uk and we will investigate on a case-by-case basis. 


\title{
Providing cancer treatment and care to people living with cancer and dementia: challenges and good practice
}

\author{
Rachael Kelley ${ }^{1}$, Claire Surr ${ }^{1}$, Alys Griffiths ${ }^{1}$, Laura Ashley ${ }^{1}$, Amanda Procter ${ }^{2}$ \\ ${ }^{1}$ Leeds Beckett University, Leeds, UK \\ ${ }^{2}$ Maggie Keswick Jencks Cancer Caring Centres Trust, Leeds, UK
}

\begin{abstract}
A growing number of people are living with cancer and dementia, creating a particular set of challenges and care needs in relation to their cancer treatment and care. These challenges exist across the cancer care pathway, from diagnosis through to decision-making, accessing treatment and care, and post-treatment, often resulting in a need for extensive support from families and further difficulties for those without familial support. This article summarises the challenges that arise when providing cancer treatment and care for people with dementia and identifies researchbased recommendations for tackling these challenges.
\end{abstract}

\section{Introduction}

In the UK there are an estimated 850,000 people living with dementia (Prince et al., 2014) and around 370,000 people newly diagnosed with cancer each year (Cancer Research UK, 2020). Both conditions are strongly associated with age; more than a third of cancer cases in the UK are in people aged 75+ (Cancer Research UK, 2020) and 7\% of all people over the age of 65 have dementia (Prince at al., 2014). The high age-related prevalence of both conditions means a considerable number of people are likely to be living with both cancer and dementia. Our recent study using UK GP records (Surr, Griffiths \& Kelley, 2020b) estimates that $7.5 \%$ of cancer patients aged $75+$ have dementia; a figure that is likely to be an underestimation due to under-diagnosis of dementia (UK diagnosis rates are around 64\% (NHS Digital, 2020). A progressively aging population, alongside improvements in cancer survival rates, means oncology staff will be required to care for increasing numbers of people living with both cancer and dementia.

Dementia is a condition with a range of effects that are likely to make cancer treatment and care more challenging. These effects, which are individual to each person, include impairments in memory, judgment, reasoning ability, language, visual perception, wayfinding and managing daily tasks, often leading to the person requiring support from family and friends. We know that people with dementia receive less cancer treatment and experience more treatment complications than patients without dementia (McWilliams, 2020; Pilleron et al., 2019), although the reasons for this are not fully understood. Potential difficulties in accessing cancer care for people with dementia include difficulties with: attending multiple oncology services and appointments; understanding information about cancer diagnoses and treatment; making treatment decisions; and undergoing treatment. Coupled with the growing prevalence of both conditions, these complex and individual needs highlight the importance of oncology services and staff knowing how to effectively provide care and support for people living with dementia and their families.

Until recently, little research has been undertaken on people living with both conditions, meaning very little was known about the experiences and challenges of accessing cancer care for people living with dementia, or how to provide them with effective cancer care and support (Hopkinson et al, 2016). Over the last few years, several UK-based studies have begun to explore these issues, and we 
now have an emerging evidence-base from which to begin improving cancer care for people living with dementia. This article reviews the emerging UK-based evidence around challenges and best practice in providing cancer nursing care and support to people living with dementia and their families, including findings from the largest and most recent UK study on this topic, undertaken by the authors (Ashley et al., 2020). We begin by setting out some of the challenges that arise when providing cancer care and support for people with dementia, before identifying research-based recommendations for tackling these challenges from recent research studies conducted in the NHS.

\section{Awareness of dementia in cancer services}

\section{The challenges}

A vital first step in addressing the needs of people with cancer who also have dementia is knowing that someone has dementia. Several studies have, however, found that oncology staff can be unaware when someone has dementia or memory problems (Courtier et al., 2016; McWilliams et al., 2018a; Martin et al, 2019; Ashley et al., 2020; Hopkinson et al., 2020). Documentation of dementia diagnoses in oncology records is inconsistent (Ashley et al., 2020; Courtier et al., 2016) and many oncology assessment forms and staff do not ask about memory problems (Ashley et al., 2020; 4Griffiths et al., 2020; Courtier et al., 2016), possibly because the prevalence of dementia-related memory difficulties and benefits of their identification for patient care are not yet well recognised amongst oncology staff (Courtier et al., 2016). During time-limited clinic appointments involving information many patients may struggle to process, it may not become apparent that someone has dementia (Ashley et al., 2020; Courtier et al., 2016; Hopkinson et al., 2020) or the person or their family may compensate for, and thus mask, the person's difficulties (Courtier et al., 2016; Hopkinson et al., 2020). In addition, people with dementia or their relatives (on whom disclosure is often reliant) may not disclose a dementia diagnosis to oncology staff (Courtier et al., 2016; Hopkinson et al., 2020) to avoid adversely affect treatment decisions, embarrassing the person, or providing potentially irrelevant information (Solomons et al., 2013), may presume this information has already been conveyed (Kelley et al., 2019), or the person's dementia or memory difficulties may not have been formally diagnosed. As a result, someone's cognitive status may not be known ahead of their appointment (McWilliams et al., 2018a) or at all (Courtier et al., 2016), reducing opportunities to ask about and meet the person's needs, or to do so in a timely manner (Courtier et al., 2016; McWilliams et al., 2018a). Diagnostic investigations and diagnoses may also be delayed due to a lack of awareness and recognition of dementia (McWilliams et al., 2018a).

In addition to difficulties identifying dementia, the majority of oncology staff report that they have had little or no training in how to identify, communicate with or support people with dementia (Ashley et al., 2020; Courtier et al., 2016). Many staff report a lack of awareness of the prevalence of memory difficulties amongst oncology patients, and of the potential benefits of identifying memory difficulties for improving patient care (Courtier et al., 2016;). Several studies have highlighted a need for dementia training for oncology staff (Courtier et al., 2016; McWilliams et al., 2018a, Ashley et al., 2020). Whilst inpatient general hospital services have undertaken initiatives to improve dementia awareness and training amongst their staff, these do not yet appear to have consistently transferred across to oncology departments, and to outpatient services in particular (Ashley at al., 2020).

\section{Research-based practice recommendations}

- Routinely ask about memory problems at initial appointments and on referral and assessment forms to enable staff to identify and meet the person's needs (Ashley et al., 
2020; Courtier et al., 2016; McWilliams et al., 2018a); consider replicating the simple memory screening questions used by many Trusts in their inpatient settings.

- Clearly record dementia or memory problems in oncology records, along with brief details of how the individual is affected and what support they may need (Ashley et al., 2020).

- Ensure oncology staff have access to good quality information and training on dementia to enable staff to better identify and support people with memory problems or dementia, and to recognise the importance of doing so (Courtier et al., 2016; McWilliams et al., 2018a; Ashley et al., 2020; Martin et al., 2016; Hopkinson et al., 2020).

\section{Making treatment decisions}

\section{The challenges}

Making decisions about cancer treatments can be particularly challenging when someone also has dementia (Griffiths et al., 2020). Although cancer treatment decisions often require careful consideration (Schiphorst et al., 2016) and many patients experience difficulties understanding treatment options or prognosis (Courtier et al., 2016), dementia brings many additional complexities. Comprehension and decision-making abilities may be affected (Zafar et al., 2009). Some people with dementia will be able to understand and make informed decisions, whereas others may require support to understand and retain information about treatment options and to make decisions, or may require other people, often family members, to do this on their behalf (Courtier et al., 2016; Witham et al., 2018; McWilliams et al., 2018a; McWilliams et al., 2018b; Griffiths et al., 2020). These difficulties are heightened if the volume and complexity of information that can be conveyed during oncology appointments is not delivered in ways that are appropriate to the communication needs and understanding of the person (McWilliams et al., 2018a; Griffiths et al., 2020; Hopkinson et al., 2020).

Prognostic uncertainty is a further challenge. Diagnostic screening and investigations may be more challenging, reducing the availability of information to inform decision-making (Torke et al., 2008), and risk-benefit judgements are particularly complex (Hopkinson et al., 2016). In addition, there is a limited evidence base to inform clinical decision-making (Hopkinson et al., 2016). For example, there is little understanding of whether cancer treatments affect dementia symptoms or medication, or of why complications from some cancer treatments are more likely for people with dementia (Hopkinson et al., 2016). Amid this general absence of evidence, decisions are often made based around how well the person is likely to cope with the treatment and its side effects and consequences (Courtier et al., 2016) - for example, the likelihood of coping well with being an inpatient post-surgery, with failing to remember why a breast has been removed (Griffiths et al., 2020) or with monitoring for treatment side-effects (Courtier et al., 2016) - and the level of the person's cognitive impairment and its impact on their life expectancy (Courtier et al., 2016; McWilliams et al., 2018a). Quantity versus quality of life becomes a key consideration, with relatives or the person often particularly keen to maintain the person's current quality of life in the context of their dementia and concerned about the potential risks of cancer treatment on the person's cognitive health and well-being (Griffiths et al., 2020; McWilliams et al., 2018b). Difficulties are commonly experienced in identifying the 'best' decision under amid an often complex and uncertain set of circumstances (Griffiths et al., 2020); with some relatives feeling this complex 'bigger picture' of dementia and other co-morbidities is not always sufficiently taken into account (Griffiths et al., 2020).

Dementia can also raise challenges and ethical dilemmas around making the 'right' decisions. This includes balancing the potentially negative impacts of imparting a cancer diagnosis multiple times due to memory problems against a desire to keep involving the person in their care (Griffiths et al., 
2020), establishing the decision-making ability of people with dementia, and determining who should make decisions (Griffiths et al., 2020). The central role played by families in decision-making is repeatedly emphasized (Courtier et al., 2016; Griffiths et al., 2020). Some, but by no means all, people with dementia are dependent on families, or on staff, to make best interest decisions on their behalf. Family members can either be informally or formally (via a Lasting Power of Attorney) appointed to help make decisions on behalf of a person who lacks capacity to make a decision (BMA, 2007); their role being to understand the preferences of the person and help determine which treatments are in their best interests. This raises several issues, including the need to ensure both family members and the person understand treatment options, the need to balance the involvement of families and the person with dementia, and the need to be alert for the possibility of differing perspectives (Harrison-Dening et al., 2016) and power imbalances influencing decision-making (Zafar et al., 2009). These power imbalances, and decision-making in general, can be particularly complex for people who lack capacity (Feinberg \& Whitlach, 2002; Kelley et al., 2020; Griffiths et al., 2020). For example, seeking treatment consent is likely to be more complex when someone lacks capacity (McWilliams et al., 2018b) and oncology staff may not recognise when someone is unable to provide informed consent, particularly if they are unaware that the person has dementia (Witham et al., 2018; Courtier et al., 2016). Whilst families are often relied upon to help make decisions, some families report a lack of information and support with making such complex, emotionally-charged decisions (Martin et al., 2016), with some feeling excluded from decision-making (McWilliams et al., 2018a; Witham et al., 2018; Martin et al, 2016) or failing to understand treatment options (McWilliams et al., 2018a). Optimising cancer care decision-making for people living with dementia is likely to be important in helping to reduce the inequalities they experience in cancer outcomes, such as increased mortality (Robb et al., 2010; Raji et al., 2008).

\section{Research-based practice recommendations}

- Treatment decision-making for people with dementia may require longer or additional appointments and repeated re-evaluation (Griffiths et al., 2020; Martin et al, 2016; McWilliams et al., 2018b).

- Information about diagnosis and treatment options should be clearly summarised and provided in easy to understand formats, such as pictures, images and short text summaries (Ashley et al., 2020; McWilliams, 2020).

- Attention should be paid to the pacing of conversations (slowing down information delivery), uncomplicated language and repetition of key information to aid recall (McWilliams et al., 2018a; McWilliams et al., 2018b; Griffiths et al., 2020). Repetition of information should be balanced on an individual basis against the potential to cause distress if a cancer diagnosis is repeatedly given 'anew' (Griffiths et al., 2020).

- Conversations should involve the person living with dementia as much as is possible, with careful attention to non-verbal body language and a warm, relaxing approach (McWilliams, 2020).

- Careful balancing of views of people with dementia and their family, and differing perspectives within families, may be required to ensure all perspectives are heard and considered (Harrison-Dening et al., 2016; Griffiths et al., 2020; Surr et al., 2020a).

- Consideration of treatment options within the 'bigger picture' context of the person's dementia and any co-morbidities and how these may, or may not, impact responses to cancer treatment and side-effects (Griffiths et al., 2020).

- Oncology staff may benefit from training and support in communicating with, and assessing decision-making capacity, in people with dementia (Griffiths et al., 2020). 
- Source information from alternative sources (e.g. family members, community services or care home staff) if the person cannot recall important clinical information or collaboration between services is required (e.g. changes to treatments requiring GP or carer input)

\section{Accessing cancer treatment and care}

\section{The challenges}

People with dementia can experience challenges in getting to, during, and after oncology appointments and treatments. Navigating multiple oncology appointments, treatment rounds and settings, and the journeys to and from them, can be particularly difficult for someone with dementia (Ashley et al., 2020; Hopkinson et al., 2020). Wayfinding to and from transport drop off points into the correct building, and then to the correct department, within large, busy, noisy and unfamiliar hospital buildings is often challenging (Ashley et al., 2020; Surr et al., 2020b). In addition, oncology departments typically lack 'dementia-friendly' designs to aid navigation; features of which include clear written and pictorial signage, use of colour and distinguishing features to aid wayfinding, good lighting; removal of unnecessary equipment and provision of stimulating activities (Moyle et al., 2008) and could help people with dementia, as well as other patients, to navigate and spend time in these spaces. Lengthy periods of waiting, for appointments and for transport, can also be difficult for people with dementia who may become frustrated, or forget where they are or why they are waiting, and attempt to leave (Ashley et al., 2020).

Providing the individualised, person-centred care that people with dementia need during appointments in oncology services can be difficult due to the involvement of multiple departments and staff (often located at different sites), a large volume of patients, and tight, protocol-driven treatment schedules (Ashley et al., 2020). People with dementia may struggle with the practicalities involved during appointments and treatments, such as dressing and un-dressing (Surr et al., 2020a), undertaking pre-treatment tasks such as drinking sufficient fluids or self-administering enemas, and understanding complex treatment instructions (Ashley et al., 2020). Recognising and reporting pain may also be more difficult; people with dementia and cancer are less likely to proactively report any pain they were experiencing, and were less likely to receive analgesia (Hopkinson et al., 2016). Impacts such as these on treatment provision for people with dementia are not always recognised by oncology staff and there may be limited consideration of how treatment pathways could be adapted to meet the person's needs (Witham et al., 2018). Inpatient oncology wards also need to be aware of the many challenges people living with dementia can face on hospital wards, including difficulties with communication, eating and drinking, distress and disorientation (Bridges et al., 2010; Kelley et al., 2019) and strategies for improving hospital care for this patient group (e.g. Handley et al., 2017; Moyle et al., 2008).

In addition to the support required before and during appointments, many people with dementia need support between treatments and appointments with managing and monitoring cancer symptoms and treatment side-effects, with undertaking self-care tasks, and with retaining and understanding information given during appointments (Surr et al., 2020a; Courtier et al., 2016). Assumptions can be incorrectly made that people with dementia will be able to independently manage care tasks once back at home, such as independently managing catheters or undertaking follow up tests and appointments (Surr et al., 2020a). The practical and emotional burden of navigating multiple appointments and tasks can be compounded if the person is simultaneously navigating care for other comorbidities (Ashley at al., 2020), which is commonly the case for people with dementia (Bunn et al., 2014). Under such circumstances, clear communication between 
professionals dealing with each condition is important to provide coordinated care and minimise confusion, but does not always happen in practice (Ashley et al., 2020).

\section{Research-based practice recommendations}

- Offer continuity of staff and environments (e.g. treatment and waiting rooms), wherever possible, to foster relationship building, aid recall, provide a sense of familiarity and reduce anxiety and unnecessary repetition of information (Ashley et al., 2020; McWilliams et al., 2018a; Witham et al., 2018; McWilliams, 2020).

- Offer pre-treatment visits and/or photos, diagrams or brief videos of departments and equipment to reduce anxiety and aid familiarity (Ashley et al., 2020; McWilliams, 2020)

- Familiarity, rapport and more effective support can also be facilitated via a simple tool for collecting information about the person's needs (Courtier et al., 2016), such as the 'This is me' tool (Alzheimer's Society, 2020), adapted for outpatient services if required.

- Offer flexibility in timing and location of appointments, including the option for telephone appointments, where possible (Ashley et al., 2020; McWilliams, 2020)

- Consider providing additional time and support during appointments and treatment sessions (Martin et al., 2016) and how treatment pathways and regimes could be adapted to meet the needs of people with dementia (Witham et al., 2018).

- Draw on existing guidance on dementia-friendly hospital environments (e.g. The King's Fund, 2020) to improve oncology departments environments - e.g. clear signage, use of colour and features to distinguish different areas, and clear display of clocks and calendars (Ashley et al., 2020) - features likely to help all patients to navigate and spend time in these spaces.

- Provide dementia-friendly books, games and activities (e.g. reminiscence materials) in waiting areas (Ashley et al., 2020)

- Offer opportunities to practice self-care tasks before leaving the hospital (Courtier et al, 2016)

\section{Involving families of people with dementia}

\section{The challenges}

A further challenge for oncology staff is involving and supporting families of people living with dementia as well as the person. Multiple studies have highlighted the many crucial roles families play in practically, cognitively and emotionally supporting people with dementia through cancer treatment and care (Surr et al., 2020a; McWilliams et al., 2018a; 2018b; Courtier et al., 2016; Martin et al., 2016; Hopkinson et al., 2020). For example, families can enable attendance and provide reassurance at appointments, monitor and report symptoms and side effects, relay information between patients and staff, and facilitate more effective consultations and care (Surr et al., 2020a; Courtier et al., 2016; McWilliams et al., 2018b; Hopkinson et al., 2020) alongside their roles in decision-making, as described earlier. Families who know the person well can be very well placed to act as advocates for the person, representing their voice if the person is not able to (Courtier et al., 2016; McWilliams et al., 2018b) and ensuring more effective consultations and care (Courtier et al., 2016).

These many challenging roles can place considerable burden on families, which may not always be considered by oncology staff or raised by families during treatment discussions (McWilliams et al., 2018a; Courtier et al., 2016). As a result, families' support needs may be overlooked by oncology services (Courtier et al., 2016; Surr et al., 2020a) despite those services relying heavily on families to 
support people with dementia to navigate cancer treatment and care (Surr et al., 2020a; Courtier et al., 2016). Adverse effects of these cumulative burdens on families include stress, worry and guilt (Surr et al., 2020a), compounded by efforts to simultaneously navigate the person's dementia journey with potentially limited support from stretched dementia services (Surr et al., 2020a), alongside one or more additional comorbidities (Nelis et al., 2019). In addition, other support avenues (such as cancer charities) may struggle to meet the specific support needs of people with dementia and their families (Surr et al., 2020a).

It is also important to note that not everyone will have a family member to accompany them to hospital appointments (Surr et al., 2020a). When there are no family members able or available to provide support, accessing cancer treatment and care can be particularly difficult for people with dementia. Unaccompanied people can experience difficulties with all of the aspects of care described above (Witham et al., 2018), resulting in missed or compromised appointments and care, distress, and additional complexity for patients and staff (Ashley et al., 2020; Witham et al., 2018; Surr et al., 2020a). In such circumstances it is often unclear who should assume responsibility for providing the significant additional support someone with dementia may need (Ashley et al., 2020). The need for staff support for unaccompanied people may not be recognised or planned for in staffing, despite often being essential to the person receiving treatment, and is instead reliant on individual staff recognising the person's additional needs and managing to flex their workload to support them (Surr et al., 2020a). In the absence of family supporters or any services able to replicate their support, other caregivers (such as bank staff or students from hospitals or care homes) may be relied upon to support the person, but can experience difficulties if they do not know the person well enough to provide effective information and support (McWilliams et al., 2018a; Surr et al., 2020a). Without sufficient support, the person's ability to undergo cancer treatment may be severely compromised (Surr et al., 2020a).

\section{Research-based practice recommendations}

- Assess the support network available to people with cancer and dementia, to determine who may be able to provide support and what support they may require to undertake this role, and to identify people who do not have access to family support (Surr et al., 2020a) to ensure their needs are addressed.

- Identify approaches for supporting people with dementia and cancer who do not have access to family support (Surr et al., 2020a) such as hospital volunteers or pre-planned support from staff

- Include families in discussion and provision of information; consider sending a nominated family member copies of hospital letters and ensure families understand and are able to support any self-care tasks required at home (Ashley et al., 2020; McWilliams, 2020)

- Involve families, if required and all parties are agreeable to this, as 'relayers of information' between staff and patients (McWilliams et al., 2018a; 2018b), including the person's known or likely preferences regarding decision-making, if the person is not able to convey these.

- Strengthen support for families of people with dementia given their crucial and often challenging roles in decision-making and enabling cancer treatment (Courtier et al., 2016; Martin et al., 2016; Ashley at al., 2020; Surr et al., 2020a; Griffiths et al., 2020)

- Enable family carers to be present during treatment, where possible, to provide reassurance and breakdown complicated instructions (McWilliams et al., 2018b; Ashley et al., 2020).

\section{Conclusion}


Dementia complicates cancer care in a range of ways accumulating across the cancer pathway. However, there are a range of strategies and support, for patients, families and staff, which can help to reduce this complexity and address the challenges that people living with dementia and their families face when accessing cancer care. The vital role many families play in enabling people with dementia to access cancer care requires greater recognition and support from cancer services, as does attention to supporting people who are unaccompanied. In recognition of the growing need to provide support for people with cancer and dementia, training, guidance and support for people with these co-morbidities has begun to be developed (see Box 1). Further work is needed to establish recognised practice guidelines for the management of cancer in people living with dementia (Hopkinson et al, 2016).

\section{Box 1 about here}

\section{Box 1: Cancer and dementia specific resources}

Macmillan provide information for people who have cancer and dementia, and those supporting them: [https://www.macmillan.org.uk/cancer-information-and-support/supportingsomeone/cancer-and-dementia]

Macmillan have also begun offering cancer-specific dementia training for oncology staff

The Society and College for Radiographers have recently produced new guidance for caring for people with dementia in radiography services: [https://www.sor.org/news/scor-update-dementiacare-guidelines]

In partnership with the Alzheimer's Society, a member of our research team has established an online forum providing peer support for people living with cancer and dementia and their families alongside expert $Q \& A$ sessions from health professionals:

[https://forum.alzheimers.org.uk/forums/caring-for-a-person-with-dementia-andcancer.81/? ga=2.55787566.48853787.1596053312-453310375.1486554788]

\section{References}

Alzheimer's Society. (2020) This is me leaflet: A support tool to enable person-centred care. https://www.alzheimers.org.uk/get-support/publications-factsheets/this-is-me [Accessed: July 2020].

Ashley, L. et al., (2020) Understanding and identifying ways to improve hospital-based cancer treatment and care for people with dementia: an ethnographic study. Age and Ageing. Under review.

BMA. (2007) Advance decisions and proxy decision-making in medical treatment and research. London: British Medical Association.

Bridges, J. Flatley, M. \& Meyer, J. (2010) Older people's and relatives' experiences in acute care settings: Systematic review and synthesis of qualitative studies. International Journal of Nursing Studies. 47: 89-97. 
Bunn, F. Burn, A. Goodman, C. Rait, G. Norton, S. Robinson, L. Schoeman, J. \& Brayne, C. (2014) Comorbidity and dementia: A scoping review of the literature. BMC Medicine. 12: 192. http://www.biomedcentral.com/1741-7015/12/192

Cancer Research UK. (2020) https://www.cancerresearchuk.org/health-professional/cancerstatistics/incidence\#heading-Two [Accessed: July 2020].

Courtier, N. Milton, R. King, A. Tope, R. Morgan, S. \& Hopkinson, J. (2016) Cancer and dementia: an exploratory study of the experience of cancer treatment in people with dementia. Psycho-oncology. 25(9): 1079-1084.

Feinberg, L.F. \& Whitlatch, C.J. (2002) Decision-making for persons with cognitive impairment and their family caregivers. American Journal of Alzheimer's Disease and Other Dementias. 2002;17(4): 237-244.

Griffiths, A. Ashley, L. Kelley, R. Cowdell, F. Collinson, M. Mason, E. Farrin, A. Henry, A. Inman, H. \& Surr, C. (2020) Decision-making in cancer care for people living with dementia. Psycho-oncology. DOI: 10.1002/pon.5448.

Handley, M. Bunn, F. \& Goodman, C. Dementia-friendly intrventions to improve the care of people living with dementia admitted to hospitals: A realist review. BMJ Open. 7:e015257. doi:10.1136/ bmjopen-2016-015257

Harrison-Dening, K. King, M. Jones, L. Vickestaff, V. \& Sampson, E.L. (2016) Advance care planning in dementia: do family carers know the treatment preferences of people with early dementia? PLOS ONE. 11(7): e0159056.

Hopkinson, J. King, A. Courtier, N. Milton, R. \& Elias, J. (2020) Potential for identification of memory problems in the cancer clinic to enable improved treatment experience and outcomes: Mixed methods case study research. European Journal of Oncology Nursing. doi:

https://doi.org/10.1016/j.ejon.2020.101777.

Hopkinson, J.B. Milton, R. King, A. \& Edwards, D. (2016) People with dementia: what is known about their experience of cancer treatment and cancer treatment outcomes? A systematic review. Psychooncology. 25(10): 1137-1146.

Kelley, R. Godfrey, M. \& Young, J. (2019) The impacts of family involvement on general hospital care experiences for people living with dementia: An ethnographic study. International Journal of Nursing Studies. 96, 72-81.

Martin, C. Shrestha, A. Burton, M, Collins, K. \& Wyld, L. (2019) How are caregivers involved in treatment decision-making for older people with dementia and a new diagnosis of cancer? Psychooncology. 28(6): 1197-1206.

McWilliams, L. (2020) An overview of treating people with comorbid dementia: Implications for cancer care. Clinical Oncology. In press.

McWilliams, L. Farrell, C. Keady, J. et al. (2018a) Cancer-related information needs and treatment decision-making experiences of people with dementia in England: a multiple perspective qualitative study. BMJ Open. 8(4): e020250. 
McWilliams, L. Swarbrick, C. Yorke, J. et al. (2018b) Bridging the divide: the adjustment and decisionmaking experiences of people with dementia living with a recent diagnosis of cancer and its impact on family carers. Ageing and Society. 1-22.

Moyle, W. Olorenshaw, R. Wallis, M. \& Borbasi, S. (2008) Best practice for the management of older people with dementia in the acute care setting: A review of the literature. International Journal of Older Peoples' Nursing. 3(2): 121-130.

Nelis, S.M. Wu, Y.T. Matthews, F.E. et al. (2019) The impact of co-morbidity on the quality of life of people with dementia: findings from the IDEAL study. Age and Ageing. 48: 316-7.

NHS Digital (2020) https://digital.nhs.uk/data-and-information/publications/statistical/recordeddementia-diagnoses/may-2020 [Accessed: July 2020].

Pilleron, S. Sarfati, D. Janssen-Heijnen, M. et al. (2019) Global cancer incidence in older adults, 2012 and 2035: a population-based study. International Journal of Cancer. 144: 49-58.

Prince, M. Knapp, M. Guerchet, M. McCrone, P. Prina, M. Comas-Herrera, A. et al. (2014) Dementia UK: Update. 2nd Edition.

Raji, M. Kuo, Y-F. Freeman, J. \& Goodwin, J. (2008) Effect of a dementia diagnosis on survival of older patients after a diagnosis of breast, colon, or prostate cancer: Implications for cancer care. Archives of Internal Medicine. 168: 2033-2040.

Robb, C. Boulware, D. Overcash, J. \& Extermann, M. (2010) Patterns of care and survival in cancer patients with cognitive impairment. Critical Reviews in Oncology/Haematology. 74: 219-224.

Schiphorst, A.H. Ten Bokkel Huinink, D. Breumelhof, R. Burgmans, J.P. Pronk, A. \& Hamaker, M.E. (2016) Geriatric consultation can aid in complex treatment decisions for elderly cancer patients. European journal of cancer care. 25(3):365-70.

Solomons, L. Solomons, J. \& Gosney, M. (2013) Dementia and cancer: A review of the literature and current practice. Aging Health. 9(3): 307-319.

Surr, C. A Griffiths, A.W. \& Kelley, R. (2019) The cancer care needs and experiences of people living with dementia [webinar]. Available at: http://www.yhscn.nhs.uk/mental-health-clinic/Dementia.php [Accessed: July 2020].

Surr, C. A. Kelley, R. Griffiths, A.W. Ashley, L. Cowdell, F. Henry, A. Collinson, M. Mason, E. \& Farrin, A.J. (2020a) Enabling people with dementia to access and receive cancer treatment and care: The crucial role of supportive networks. Journal of Geriatric Oncology.

https://doi.org/10.1016/i.jgo.2020.03.015

Surr, C.A. Griffiths, A.W. Kelley, R. Ashley, L. Cowdell, F. Henry, A. Inman, H. Collinson, M. Mason, E. \& Farrin, A. (2020b) Navigating cancer treatment and care when living with comorbid dementia: An ethnographic and interview study. Under review.

The King's Fund. (2020) Developing supportive design for people with dementia: Enhancing the healing environment. https://www.kingsfund.org.uk/projects/enhancing-healing-environment/ehedesign-dementia [Accessed: July 2020]. 
Torke, A.M. Schwartz, P.H. Holtz, L.R. Montz, K. \& Sachs, G.A. (2013) Caregiver perspectives on cancer screening for persons with dementia: "Why put them through it?". Journal of the American Geriatrics Society. 61(8): 1309-1314.

Witham, G. Haigh, C. Mitchell, D. \& Beddow, A. (2018) Carer experience supporting someone with dementia and cancer: a narrative approach. Qualitative Health Research. 28 (5): 813-823.

Zafar, S.Y. Alexander, S.C. Weinfurt, K.P. Schulman, K.A. \& Abernethy, A.P. (2009) Decision making and quality of life in the treatment of cancer: a review. Supportive Care in Cancer. 17(2): 117-27. 